\title{
New constraints on the average escape fraction of Lyman continuum radiation in $z \sim 4$ galaxies from the VIMOS Ultra Deep Survey (VUDS) ${ }^{\star}$
}

F. Marchi ${ }^{1}$, L. Pentericci ${ }^{1}$, L. Guaita ${ }^{1}$, B. Ribeiro ${ }^{2}$, M. Castellano ${ }^{1}$, D. Schaerer ${ }^{3,7}$, N. P. Hathi ${ }^{2,11}$, B. C. Lemaux $^{10}$, A. Grazian ${ }^{1}$, O. Le Fèvre ${ }^{2}$, B. Garilli ${ }^{6}$, D. Maccagni ${ }^{6}$, R. Amorin ${ }^{8,9}$, S. Bardelli ${ }^{4}$, P. Cassata ${ }^{5}$, A. Fontana ${ }^{1}$, A. M. Koekemoer ${ }^{11}$, V. Le Brun ${ }^{2}$, L. A. M. Tasca ${ }^{2}$, R. Thomas ${ }^{2}$, E. Vanzella ${ }^{4}$, G. Zamorani ${ }^{4}$, and E. Zucca ${ }^{4}$

1 INAF-Osservatorio Astronomico di Roma, via Frascati 33, 00040 Monte Porzio Catone, Italy e-mail: francesca.marchi@oa-roma.inaf.it

2 Aix Marseille Université, CNRS, LAM (Laboratoire d'Astrophysique de Marseille) UMR 7326, 13388 Marseille, France

3 Geneva Observatory, University of Geneva, ch. des Maillettes 51, 1290 Versoix, Switzerland

4 INAF-Osservatorio Astronomico di Bologna, via Ranzani, 1, 40127 Bologna, Italy

5 Instituto de Fisica y Astronomía, Facultad de Ciencias, Universidad de Valparaíso, Gran Bretaña 1111, Playa Ancha, Valparaíso, Chile

${ }^{6}$ INAF-IASF Milano, via Bassini 15, 20133 Milano, Italy

${ }^{7}$ Institut de Recherche en Astrophysique et Planétologie - IRAP, CNRS, Université de Toulouse, UPS-OMP, 14 avenue É. Belin, 31400 Toulouse, France

${ }^{8}$ Cavendish Laboratory, University of Cambridge, 19 JJ Thomson Avenue, Cambridge, CB3 OHE, UK

9 Kavli Institute for Cosmology, University of Cambridge, Madingley Road, Cambridge CB3 OHA, UK

10 Department of Physics, University of California, Davis, One Shields Ave., Davis, CA 95616, USA

11 Space Telescope Science Institute, 3700 San Martin Drive, Baltimore, MD 21218, USA

Received 14 November 2016 / Accepted 23 December 2016

\section{ABSTRACT}

Context. Determining the average fraction of Lyman continuum $(\mathrm{LyC})$ photons escaping high redshift galaxies is essential for understanding how reionization proceeded in the $z>6$ Universe.

Aims. We want to measure the LyC signal from a sample of sources in the Chandra Deep Field South (CDFS) and COSMOS fields for which ultra-deep VIMOS spectroscopy as well as multi-wavelength Hubble Space Telescope (HST) imaging are available.

Methods. We select a sample of 46 galaxies at $z \sim 4$ from the VIMOS Ultra Deep Survey (VUDS) database, such that the VUDS spectra contain the LyC part, that is, the rest-frame range 880-910 А. Taking advantage of the HST imaging, we apply a careful cleaning procedure and reject all the sources showing nearby clumps with different colours, that could potentially be lower-redshift interlopers. After this procedure, the sample is reduced to 33 galaxies. We measure the ratio between ionizing flux (LyC at $895 \AA$ ) and non-ionizing emission (at $\sim 1500 \AA$ ) for all individual sources. We also produce a normalized stacked spectrum of all sources.

Results. Assuming an intrinsic average $L_{v}(1470) / L_{v}(895)$ of 3 , we estimate the individual and average relative escape fraction. We do not detect ionizing radiation from any individual source, although we identify a possible LyC emitter with very high Ly $\alpha$ equivalent width (EW). From the stacked spectrum and assuming a mean transmissivity for the sample, we measure a relative escape fraction $f_{\mathrm{esc}}^{\mathrm{rel}}=0.09 \pm 0.04$. We also look for correlations between the limits in the LyC flux and source properties and find a tentative correlation between LyC flux and the EW of the Ly $\alpha$ emission line.

Conclusions. Our results imply that the LyC flux emitted by $V=25-26$ star-forming galaxies at $z \sim 4$ is at most very modest, in agreement with previous upper limits from studies based on broad and narrow band imaging.

Key words. galaxies: high-redshift - galaxies: evolution - galaxies: star formation

\section{Introduction}

Studying the process of hydrogen reionization in the early Universe and identifying the sources of UV radiation responsible for this process are amongst the most challenging tasks of modern extra-galactic astronomy. The radiation able to ionize the neutral hydrogen falls at wavelengths shorter than $912 \AA$ (Lyman continuum, LyC) and is produced by massive OB-type stars in young star clusters and by active galactic nuclei (AGN).

\footnotetext{
* Based on data obtained with the European Southern Observatory Very Large Telescope, Paranal, Chile, under Large Program 185.A-0791.
}

Therefore, star-forming galaxies and AGN at $z \sim 6-7$ are most likely responsible for this phenomenon (Robertson et al. 2015; Giallongo et al. 2015). However, it is extremely difficult to directly measure their contribution since at redshift higher than $z=5$, the intergalactic medium (IGM) becomes completely opaque to LyC photons and prevents the direct detection of Lyman continuum flux (Inoue et al. 2014; Worseck et al. 2014). In some cases it could be possible to detect $\mathrm{LyC}$ emission at higher redshifts if the object is very bright in LyC and resides in a particular line of sight that favours the escape of LyC photons. These are, however, particular cases that are not able to give us statistics on the populations of LyC emitters at high redshifts. 
We therefore rely on the study of $z<4.5$ galaxies to understand the physical properties of the objects emitting LyC radiation and later infer if these properties are more common during the reionization epoch. The LyC radiation from galaxies between redshifts of 2.5 and 4.5 can, in principle, be detected from the ground, while for galaxies at lower redshift, we must rely on space-based observations. The ionizing radiation is attenuated by neutral gas and dust in the interstellar and circumgalactic medium of the sources. Therefore, the detection of LyC emission in individual galaxies is a rather difficult task.

There are different methods to evaluate the LyC escape fraction, that is, the fraction of HI-ionizing photons that are not absorbed by the interstellar medium (ISM) and are thus free to ionize the neutral hydrogen in the IGM. The first one relies on narrow-band photometry, that is, the study of galaxies in a given (narrow) redshift range such that the supposed LyC flux would fall precisely in a narrow band filter. With this method, the narrow band collects precisely the flux in the LyC range: however, because of the limited redshift range probed by a typical narrow band filter, only a low number of sources can be studied at the same time. Guaita et al. (2016) used this technique to analyse a sample of 67 galaxies at $z \sim 3$ (some also from VUDS), finding no individual detections and an upper limit on the relative escape fraction of ionizing photons of $12 \%$ for the entire sample. Mostardi et al. (2015) also applied the narrow band technique to a sample of $16 z \sim 3$ Lyman-break-selected star-forming galaxies (LBG) and narrow-band-selected Lyman- $\alpha$ emitters, finding one LyC emitter candidate among the LBGs at $z=3.14$ with a relative escape fraction of $f_{\mathrm{esc}}^{\mathrm{rel}} \sim 75-100 \%$. Finally, earlier studies by Iwata et al. (2009) identified several candidate emitters amongst the LAE (125 galaxies) and LBG (73 galaxies) populations of the SSA22 protocluster region at $z=3.09$.

An alternative method consists of using broad-band imaging to capture the LyC flux. In this way, one can simultaneously study a much larger number of galaxies in a broader redshift range. The drawback of this technique resides in the fact that the filter contains both LyC and non-LyC flux from the source and therefore a more careful analysis is needed to disentangle the two components. Grazian et al. (2016) and Boutsia et al. (2011) applied this technique on samples (37 and 11 galaxies, respectively) of $z \sim 3$ star-forming galaxies with secure redshifts, finding no significant detection. They used a relatively narrow redshift range so that the $U$ filter on LBC would sample only the LyC radiation, and found average upper limits of $f_{\text {esc }}^{\text {rel }} \lesssim 2 \%$ and $f_{\text {esc }}^{\text {rel }} \lesssim 5 \%$, respectively.

Finally, it is also possible to directly analyse spectral observations and measure the flux in the LyC region of the spectrum. Fewer galaxies have deep enough spectroscopic observations compared to those with photometric ones. Giallongo et al. (2002), Steidel et al. (2001), Shapley et al. (2006), Bogosavljevìc (2010) and Shapley et al. (2016) used this method to evaluate the escape fraction of high-redshift galaxies finding several LyC emitter candidates.

All the above methods are affected by the problem of line of sight $(\mathrm{LoS})$ contamination that is the main limitation of LyC studies when imaging and spectroscopic observations are taken from the ground (Vanzella et al. 2012). Indeed lowredshift galaxies can mimic the LyC emission from high-redshift sources if they are located very close to the target galaxies and the spatial resolution does not allow us to distinguish them. These nearby contaminants can only be identified in highresolution Hubble Space Telescope (HST) images and appear blended in ground-based observations. In most cases, the putative LyC emission appears in HST images offset with respect to the main optical galaxy (Nestor et al. 2011; Mostardi et al. 2013), indicating the presence of a possible lower-redshift contaminant. To date, there are only two galaxies with an unambiguous LyC detection at high redshifts. The first one is the object Ion2, a very compact star-forming galaxy at $z=3.212$, detected by Vanzella et al. (2015) from deep imaging. It was analysed in detail by de Barros et al. (2016) and confirmed by Vanzella et al. (2016). A second clear Lyman continuum emitter was recently identified at $z=3.15$ by Shapley et al. (2016). They used the spectroscopic technique combined with high-resolution HST imaging and inferred a value of $f_{\text {esc }} \geq 42 \%$ at $95 \%$ confidence for this source.

In this work, we study the LyC emission of a sample of galaxies at $z \sim 4$ using ultra-deep spectroscopic observations from the VUDS collaboration (Le Fèvre et al. 2015) paying particular attention to minimise the possibility of having nearby lower-redshift contaminants close to our targets. This can be done thanks to the availability of deep multicolour HST imaging. The paper is organized as follows: in Sect. 2, we describe the sample selection; in Sect. 3, we measure the LyC emission in individual spectra and define the quantities needed to evaluate the relative escape fraction; in Sect. 4, we present the stack of the spectra and in Sect. 5, we describe the results; finally, we discuss the results in view of future spectroscopic surveys in Sect. 6. Throughout the paper we adopt the $\Lambda$ cold dark matter $(\Lambda$-CDM) cosmological model $\left(H_{0}=70 \mathrm{~km} \mathrm{~s}^{-1} \mathrm{Mpc}^{-1}, \Omega_{\mathrm{M}}=0.3\right.$ and $\left.\Omega_{\Lambda}=0.7\right)$. All magnitudes are in the AB system.

\section{Sample selection}

Our sample contains galaxies selected from the VIMOS Ultra Deep Survey (Le Fèvre et al. 2015, VUDS) database ${ }^{1}$, which is the largest spectroscopic survey of galaxies at $z>2$. VUDS acquired spectra to identify approximately 7000 galaxies at $2 \leq$ $z \leq 6$. We selected all galaxies with reliable redshifts in the range $3.5 \leq z \leq 4.5$ (see below) and HST multi-wavelength coverage. The choice of the redshift interval relies on our aim to measure a possible LyC signal in the wavelength range covered by the VUDS spectra (3800-9400 ̊). We therefore need sources at $z>3.5$ to have the LyC interval in the spectrum. The upper redshift limit is instead due to the fact that the Inter Galactic Medium is almost totally opaque at $z>4.5$ (Madau 1995; Inoue et al. 2014).

All the galaxies with VUDS reliability flags 3 and 4, corresponding to a probability greater than $95 \%$ for the spectroscopic redshift to be correct, were included in the sample. Only in the cases where the photometric redshift was consistent with the measured spectroscopic redshift, within $1 \sigma$, we also included those with flags 2 and 9, corresponding in both cases to a probability of approximately $80 \%$ for the redshift to be correct with the difference that the redshift of flag 9 objects is based only on one clear emission line in the spectrum (see Le Fèvre et al. 2015, for more details). In this procedure, we used CANDELS and MUSYC photometric redshifts (Dahlen et al. 2013; Cardamone et al. 2010). We remark here the excellent quality of the flux calibration of the VUDS spectra that, if not done properly, could significantly affect the evaluation of $f_{\mathrm{esc}}^{\mathrm{rel}}$. The spectra are calibrated using spectrophotometric standard stars with a relative flux accuracy of better than $\sim 5 \%$ over the wavelength range 3600 to $9300 \AA$. In addition, each spectrum is corrected for atmospheric extinction and for wavelength-dependent slit

http://cesam. lam. fr/vuds/DR1/ 
losses due to atmospheric refraction, taking into account the geometry of each source as projected into the slit (Thomas et al. 2017). The spectra are also corrected for the galactic extinction (Le Fèvre et al. 2015).

The last requirement in our sample selection procedure is the HST coverage: observations in at least two HST bands are necessary to identify possible foreground contaminants that can mimic the LyC emission from the target.

In total, we selected 46 galaxies with the above characteristics. Out of 46, 11 sources are in the COSMOS field and have CANDELS coverage (Koekemoer et al. 2011), whereas the remaining sources are in the Extended Chandra Deep Field South (ECDFS). Of these, 13 of the galaxies are covered by CANDELS multi-wavelength imaging (Koekemoer et al. 2011; Grogin et al. 2011) and 22 fall outside the CANDELS field but within the area covered by GEMS (Rix et al. 2004). We did not consider the sources in the COSMOS area outside CANDELS because, for them, only one HST band $(F 814 W)$ is available and it would not be deep enough for our purpose. For the sources that have CANDELS coverage, we used the HST bands ACS/F435W, $A C S / F 606 W$ and $F 814 W$ (hereafter referred to as $B, V$ and $I$ ) for ECDFS, whereas for COSMOS, we used only $V$ and $I$. Finally, for the sources covered by GEMS, we used HST/ACS F606W and HST/ACS F850LP (hereafter referred to as $V$ and $z$ ).

We checked the position of our galaxies in the CANDELS fields, in the stellar mass vs. UV absolute magnitude $M_{1400}$ plane, where $M_{1400}$ was evaluated from the $V$ band magnitude. The masses that we used are the reference masses for CANDELS. They were obtained by Santini et al. (2015) combining, with a median mass approach, different mass estimates derived with the $\mathrm{BC} 03$ templates, a Chabrier IMF and various parametrizations of the SFH with some of the models, also including nebular emission contribution. Comparing our sample to the general population of galaxies at $3.5<z<4.5$ in the GOODS-South field (Grazian et al. 2015), we conclude that it is representative of the general population of star-forming galaxies with $M_{\mathrm{UV}} \sim-20$ in that redshift range.

To avoid false LyC detection, we applied a careful cleaning procedure to our sample by removing all the galaxies with possible projected contamination on an object-by-object basis. Following Guaita et al. (2016), we proceeded with three different steps. The first consists of identifying isolated, single-component sources from those with multiple components with a direct inspection of the HST images; we then assume that the singlecomponent sources are unlikely to be contaminated by foreground galaxies. The second step consists of generating colour images for each multi-component source identified in the previous step and analysing each sub-component. To do this, we used the iraf task IMSTACK to generate colour images in $B-V-I$ bands for the galaxies in the area covered by CANDELS in the CDFS field, $V-z$ images for those covered by GEMS and $V-I$ images for the sources in COSMOS. An individual component or a region of the galaxy with appreciably different colours (see below) with respect to the main galaxy is likely to be an interloper at a different redshift. Therefore, generating these images allowed us to reject these contaminated sources from the sample (Vanzella et al. 2012; Siana et al. 2015).

Actually, the situation is slightly more complex, as multiple components (hereafter clumps) belonging to the same galaxy can be redder or bluer than the main component of the galaxy, depending on the properties of their stellar populations, dust and the presence and the strength of emission lines falling within the broad-band filters. The best way to take this into account would
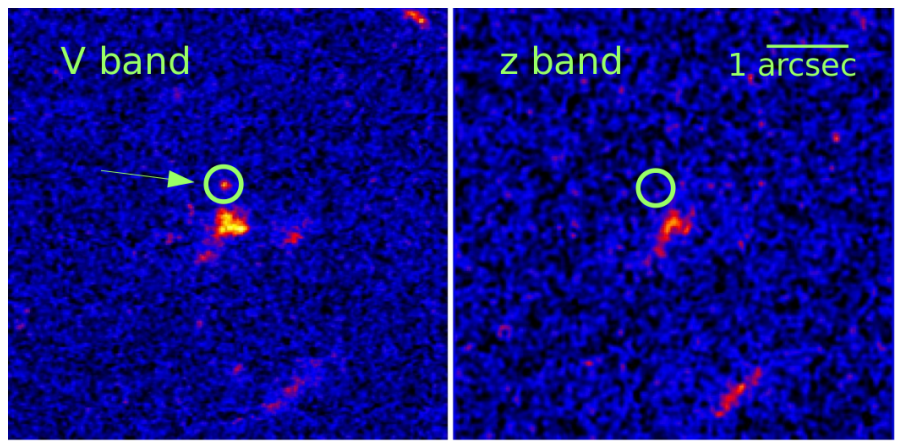

Fig. 1. Example of an object discarded in the second step of the cleaning procedure. This is a galaxy at $z=3.5953$ within GEMS coverage. In the figure, we show the two images of the source in the $V$ band and in the $z$ band. This was rejected from the sample due to the presence of a clump in the $V$ band image that is not visible in the $z$ band image.

be to perform accurate SED fittings of each individual clump of each multi-component galaxy. However, this accurate analysis is only possible when many different bands are available, as in the CANDELS fields, but would not be possible, for example, for GEMS or the entire COSMOS. In our previous work (Guaita et al. 2016), after an accurate analysis of a small sample of VUDS galaxies with similar $V$-band magnitude as our objects, we showed that simple colour cuts can give results that are entirely comparable to a full SED fitting of individual clumps. To reject sources on the basis of the colours, we thus used the following criteria: $\Delta(B-I)>0.2$ and $\Delta(V-I)>0.2$ for CANDELS and $\Delta(V-z)>0.2$ for GEMS. The colours of the clumps were determined by running Source Extractor (SExtractor, Bertin \& Arnouts 2010) on the sources: Sextractor configuration parameters for background, source extraction and optimal photometry were fixed in order to separate the individual clumps and maximise the signal-to-noise ratio.

In Guaita et al. (2016), we showed that relying on colours alone is a conservative approach because this selection tends to overestimate the number of contaminated sources. The purity of our sample is, however, our primary concern, since the presence of contaminants is the most worrying aspect in the search for LyC emitters (Vanzella et al. 2012; Mostardi et al. 2015). With the second step of the cleaning procedure, we excluded eight sources from the sample: one of these is shown in Fig. 1.

The last step of our cleaning procedure consists of directly inspecting the two-dimensional spectrum of each remaining source to look for the presence of defects in the spectral region of interest (slit-border defects, sky line residuals etc.) and to check that the spectra do not show hints of possible contamination. With this direct inspection, we rejected five further sources from the sample. Three of these show slit-border defects in the LyC region of the spectrum, whereas the other two seem to be contaminated by other sources. Both these galaxies were identified in the previous step as multi-component but were kept in the sample because they did not show a large colour difference in $V-z$. However, their two-dimensional spectra show an emission in the LyC region that is shifted in the y-axis compared to the rest, meaning that it is associated only to one component of the galaxy. Unfortunately both these galaxies are in the GEMS area where we only have two bands: it is therefore harder to determine if the clump really belongs to the galaxy or is at a different redshift. We decided to discard the two galaxies from the sample. It is worth noting that all the galaxies rejected in this 
Table 1. Number of sources in the different steps of the cleaning procedure in the three fields.

\begin{tabular}{lcccc}
\hline \hline & COSMOS/CANDELS & ECDFS/CANDELS & ECDFS/GEMS & TOTAL \\
\hline \#initial sources & 11 & 13 & 22 & 46 \\
\#sources after step 2 & 9 & 11 & 18 & 38 \\
\#sources after step 3 & 9 & 11 & 13 & 33 \\
\hline
\end{tabular}

Notes. We indicate, with ECDFS/GEMS, the area of GEMS not covered by CANDELS. "Initial sources", "sources after step 2" and "sources after step 3" indicate the number of sources before the cleaning procedure is applied, after the colour cleaning and after the spectral check, respectively.
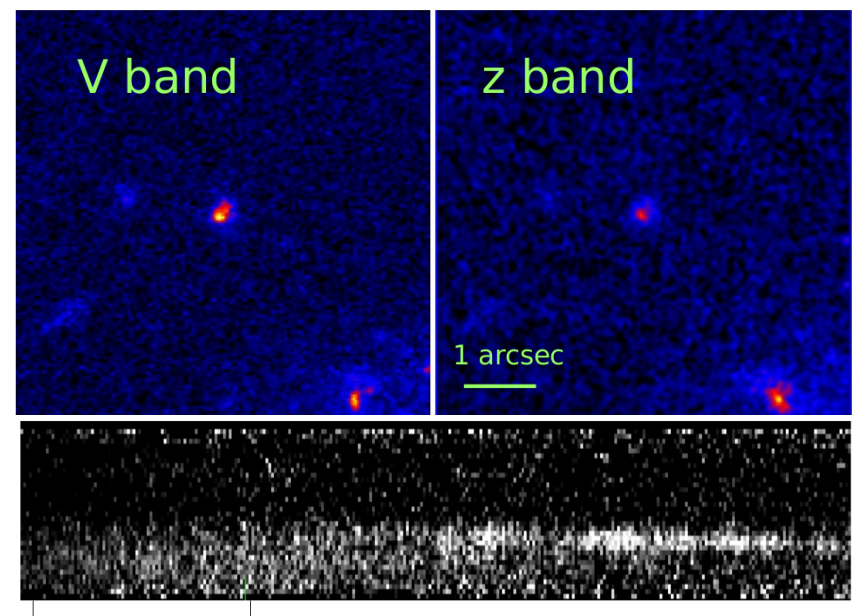

LyC

Fig. 2. Example of an object at $z=3.536$ discarded in the third step of the cleaning procedure. Upper panel: images of the source in the $V$ band and in the $z$ band. Lower panel: portion of the two-dimensional spectrum of the source that contains the LyC region that is affected by border defects.

step of the cleaning procedure are covered by GEMS for which, in the previous step, we could check only one colour. This final step is therefore complementary to the second step of the cleaning procedure for the sources in GEMS. In Fig. 2, we show an example of a source that we excluded from the sample due to the presence of slit-border defects.

Finally, we remark that, with this cleaning procedure, it is not possible to identify contaminants that are exactly superposed with the high-redshift galaxy. This is, however, very unlikely and we do not consider this particular case.

In Table 1, we show the number of galaxies in the three HST fields in the different steps of the cleaning procedure. The final sample consists of 33 galaxies and is listed in Table 2. From here on, we refer to this sample as the clean sample. In Fig. 3, we show the redshift and the $V$ magnitude distributions of the galaxies in the clean sample (semi-filled histogram) compared to those in the initial sample (empty histogram). From this comparison, it is clear that the excluded sources are a random subset of the entire sample in terms of $V$ band and redshift distribution.

\section{Measuring the LyC signal: the escape fraction of ionising photons}

The escape fraction is the fraction of Lyman continuum photons produced by massive OB-type stars in star-forming galaxies that escape from the galaxy into the IGM without being absorbed, relative to the total number produced
(Wyithe \& Cen 2007; Wise \& Cen 2009, and references therein). This quantity, known as the absolute escape fraction, $f_{\text {esc }}$, requires knowledge of the intrinsic number of ionising photons produced by the galaxy to determine it. However, the intrinsic spectral energy distribution (SED) of a galaxy is not known a priori, especially in the rest-frame far UV where dust reddening could be severe.

A related quantity, more used in observational studies, is the relative escape fraction, which is defined as (Steidel et al. 2001; Siana et al. 2007):

$f_{\mathrm{esc}}^{\mathrm{rel}}(\mathrm{LyC})=\frac{L_{v}(1470) / L_{v}(895)}{f_{v}(1470) / f_{v}(895) \cdot \mathrm{e}^{-\tau_{\mathrm{IGM}, z}}}$,

where $L_{v}(1470) / L_{\nu}(895)$ is the ratio of the intrinsic luminosities at 1470 and $895 \AA$ rest frame and $f_{v}(1470) / f_{v}(895)$ is the ratio of the observed flux densities at the same wavelengths. $\mathrm{e}^{-\tau_{\mathrm{IGM}}, z}$ is the transmissivity, a quantity that allows us to take into account the photoelectric absorption of photons with $\lambda \leq 912 \AA$ by the IGM. $f_{\mathrm{esc}}^{\text {rel }}(\mathrm{LyC})$ is a useful observational quantity because it can be defined with respect to the UV continuum luminosity, which is accessible to direct observations. For star-forming galaxies in fact, at $z \sim 4$, the continuum flux at $\lambda \sim 1470 \AA$ used in Eq. (1) can be determined from observations. It is also possible to determine $f_{\text {esc }}$ from $f_{\text {esc }}^{\text {rel }}$ if the degree of dust extinction in the UV is known: $f_{\text {esc }}$ is defined indeed as the relative escape fraction multiplied by the extinction of the dust. In the absence of dust, the two quantities are equivalent.

In the following subsections, we describe our procedures to derive the quantities in Eq. (1) to estimate the relative escape fraction of our clean sample of galaxies.

\subsection{Intrinsic $L_{v}(1470) / L_{v}(895)$ ratio and IGM transmissivity}

The intrinsic luminosity ratio $L_{v}(1470) / L_{v}(895)$ depends on the physical properties of the galaxies, such as the mean stellar ages, metallicities, stellar initial mass functions (IMFs), and star formation histories (SFHs) as also shown in Guaita et al. (2016, see Table 3); for typical star-forming galaxies at $z \sim 3$, it varies between 1.7 and 7.1 for an age between $1 \mathrm{Myr}$ and 0.2 Gyr, adopting the Bruzual \& Charlot (2003) library (Grazian et al. 2016). For easier comparison with earlier studies (Steidel et al. 2001; Grazian et al. 2016), we adopted a value of $L_{v}(1470) / L_{v}(895)=3$, which corresponds to young star-forming galaxies of $\sim 10 \mathrm{Myr}$ assuming a constant Bruzual \& Charlot SFH and a Chabrier IMF (Chabrier 2003). We checked the best fit physical parameters of the galaxies in the CANDELS fields (Santini et al. 2015) in our sample, and for most models their age is actually closer to 100-200 Myr. Therefore, a more appropriate value for the intrinsic $L_{v}(1470) / L_{v}(895)$ would be 5-6 (Guaita et al. 2016). However we note that this is simply a multiplicative factor in the evaluation of the relative escape fraction, and it is therefore possible to re-scale $f_{\mathrm{esc}}^{\mathrm{rel}}(\mathrm{LyC})$ with other values of $L_{v}(1470) / L_{v}(895)$ if needed. 
Table 2. Sources in the clean sample.

\begin{tabular}{|c|c|c|c|c|c|c|c|c|c|c|c|}
\hline (1) & (2) & (3) & (4) & (5) & (6) & (7) & (8) & (9) & (10) & (11) & (12) \\
\hline IDvuds & ID & $\begin{array}{c}\text { RA } \\
\text { (deg) }\end{array}$ & $\begin{array}{c}\text { Dec } \\
(\mathrm{deg})\end{array}$ & $z_{\text {spec }}$ & Flag & $\begin{array}{c}V \\
(\mathrm{mag}) \\
\end{array}$ & $\begin{array}{l}f_{\lambda}(895) \\
\left(10^{-19}\right) \\
\end{array}$ & $\begin{array}{c}\operatorname{err} f_{\lambda}(895) \\
\left(10^{-19}\right)\end{array}$ & $\begin{array}{c}f_{\lambda}(1470) \\
\left(10^{-19}\right) \\
\end{array}$ & $\begin{array}{c}\operatorname{err} f_{\lambda}(1470) \\
\quad\left(10^{-19}\right) \\
\end{array}$ & $\begin{array}{r}E W_{\text {Ly } \alpha} \\
(\AA) \\
\end{array}$ \\
\hline \multicolumn{12}{|c|}{ COSMOS/CANDELS } \\
\hline 510998698 & 6772 & 150.082313 & 2.261036 & 4.0651 & 9 & $25.83 \pm 0.12$ & 1.98 & 3.23 & 9.04 & 1.49 & -24.99 \\
\hline 511002138 & 4913 & 150.122450 & 2.237101 & 4.3600 & 9 & $24.90 \pm 0.06$ & 8.25 & 3.26 & 17.08 & 5.63 & -19.18 \\
\hline 511227001 & 20282 & 150.062182 & 2.423024 & 3.6350 & 3 & $25.57 \pm 0.11$ & 1.79 & 1.39 & 9.26 & 0.48 & -23.84 \\
\hline 5100998496 & 6868 & 150.205055 & 2.262162 & 3.8979 & 4 & $25.90 \pm 0.15$ & -3.93 & 1.68 & 17.24 & 0.60 & $\geq 0$ \\
\hline 5101226001 & 20579 & 150.189291 & 2.427818 & 3.7327 & 3 & $25.71 \pm 0.11$ & 1.39 & 2.10 & 11.70 & 0.46 & $\geq 0$ \\
\hline 5101226251 & 20598 & 150.157203 & 2.42786 & 3.9888 & 3 & $25.42 \pm 0.10$ & -4.95 & 1.40 & 17.74 & 0.52 & $\geq 0$ \\
\hline $5101233433 *$ & 16703 & 150.186851 & 2.379107 & 3.7403 & 4 & $25.19 \pm 0.08$ & 5.35 & 1.64 & 18.72 & 0.38 & $\geq 0$ \\
\hline 5101233724 & 16397 & 150.177453 & 2.375223 & 4.3862 & 4 & $26.51 \pm 0.20$ & -1.73 & 1.55 & 9.55 & 0.56 & -11.11 \\
\hline 5101242274 & 11634 & 150.191107 & 2.317958 & 4.3771 & 4 & $25.95 \pm 0.16$ & -0.34 & 0.80 & 19.61 & 0.67 & $\geq 0$ \\
\hline \multicolumn{12}{|c|}{ ECDFS/CANDELS } \\
\hline 530029038 & 3753 & 53.0792917 & -27.8772595 & 4.4179 & 3 & $26.70 \pm 0.19$ & 1.27 & 2.41 & 14.79 & 0.56 & $\geq 0$ \\
\hline 530030313 & 4503 & 53.1132794 & -27.8698754 & 3.5789 & 3 & $26.04 \pm 0.09$ & 3.83 & 3.87 & 10.62 & 0.61 & $\geq 0$ \\
\hline 530030325 & 4542 & 53.1090745 & -27.8697555 & 3.7519 & 4 & $25.42 \pm 0.06$ & -0.34 & 1.41 & 21.25 & 0.43 & $\geq 0$ \\
\hline 530032655 & 5955 & 53.0940950 & -27.854974 & 3.7222 & 2 & $25.71 \pm 0.07$ & -0.80 & 1.24 & 13.67 & 0.41 & $\geq 0$ \\
\hline 530036055 & 8312 & 53.2208728 & -27.8334905 & 4.1608 & 3 & $25.36 \pm 0.05$ & -1.29 & 1.21 & 27.90 & 0.85 & $\geq 0$ \\
\hline 530037593 & 9317 & 53.156571 & -27.824343 & 3.5336 & 2 & $25.79 \pm 0.08$ & -2.44 & 2.46 & 11.68 & 0.36 & $\geq 0$ \\
\hline 530047200 & 17081 & 53.0640040 & -27.765834 & 3.5600 & 2 & $26.06 \pm 0.09$ & 6.36 & 14.9 & 16.27 & 1.49 & $\geq 0$ \\
\hline $530049753^{*}$ & 18915 & 53.2104941 & -27.7502276 & 3.6055 & 4 & $25.81 \pm 0.08$ & 2.92 & 0.93 & 10.68 & 0.20 & $\geq 0$ \\
\hline 530049877 & 18722 & 53.0147863 & -27.7517345 & 3.8245 & 3 & $25.75 \pm 0.08$ & 2.53 & 2.20 & 16.14 & 0.50 & -8.43 \\
\hline 530050023 & 19009 & 53.2048527 & -27.7494405 & 3.6097 & 4 & $25.07 \pm 0.04$ & -3.43 & 2.43 & 21.29 & 0.55 & $\geq 0$ \\
\hline 530051970 & 20286 & 53.1989481 & -27.7379129 & 3.7983 & 4 & $24.91 \pm 0.04$ & -1.20 & 1.87 & 26.33 & 0.79 & -5.32 \\
\hline \multicolumn{12}{|c|}{ ECDFS/GEMS } \\
\hline 530003871 & 958 & 53.196500 & -28.036822 & 3.9022 & 3 & $26.23 \pm 0.12$ & 1.61 & 1.25 & 11.81 & 0.49 & $\geq 0$ \\
\hline 530004745 & 1087 & 53.204005 & -28.03064 & 3.6447 & 3 & $26.24 \pm 0.11$ & -3.48 & 2.8 & 12.27 & 0.66 & -18.61 \\
\hline 530008598 & 432 & 53.004328 & -28.006944 & 4.0409 & 4 & $25.04 \pm 0.04$ & 2.73 & 2.32 & 40.80 & 0.91 & -15.72 \\
\hline 530010169 & 624 & 53.209205 & -27.996984 & 3.9443 & 3 & $26.65 \pm 0.18$ & -1.41 & 1.13 & 12.59 & 0.52 & $\geq 0$ \\
\hline 530013910 & 233 & 53.222218 & -27.973808 & 3.9560 & 4 & $24.59 \pm 0.03$ & 4.21 & 1.52 & 48.19 & 0.82 & -2.76 \\
\hline 530046805 & 164 & 52.98375 & -27.768377 & 3.5748 & 4 & $25.03 \pm 0.04$ & 0.35 & 1.55 & 20.63 & 0.34 & $\geq 0$ \\
\hline 530063568 & 629 & 53.198749 & -27.666776 & 4.1005 & 4 & $26.10 \pm 0.11$ & 4.18 & 2.32 & 13.99 & 0.75 & $\geq 0$ \\
\hline 530070083 & 22 & 53.048402 & -27.626923 & 3.6377 & 3 & $24.68 \pm 0.03$ & -5.93 & 2.24 & 26.74 & 0.76 & $\geq 0$ \\
\hline 530074267 & 322 & 53.135104 & -27.601156 & 3.5125 & 3 & $25.27 \pm 0.05$ & -1.22 & 3.17 & 13.86 & 0.41 & $\geq 0$ \\
\hline $530075924 *$ & 1256 & 53.060854 & -27.590558 & 3.5707 & 3 & $25.24 \pm 0.05$ & 5.68 & 1.38 & 12.25 & 0.36 & -65.00 \\
\hline 530076805 & 1402 & 53.247191 & -27.584018 & 3.9602 & 2 & $26.64 \pm 0.18$ & -3.36 & 2.77 & 16.30 & 1.04 & -1.46 \\
\hline 535003653 & 852 & 53.080489 & -28.037964 & 4.2093 & 3 & $26.70 \pm 0.18$ & 1.95 & 0.88 & 4.26 & 0.45 & -160.39 \\
\hline 535016440 & 1576 & 53.051566 & -27.957000 & 4.3215 & 3 & $27.93 \pm 0.58$ & -0.60 & 0.88 & 5.41 & 0.37 & $\geq 0$ \\
\hline
\end{tabular}

Notes. (1) VUDS identification number; (2) CANDELS or GEMS identification number; (3, 4) right ascension and declination; (5) spectroscopic redshift; (6) redshift quality flag (see Le Fèvre et al. 2015, for details); (7) $V$ magnitude and respective error; $(8,9)$ mean flux in the wavelength range 880-910 $\AA$ and respective error in unit of $10^{-19} \mathrm{erg} \mathrm{s}^{-1} \mathrm{~cm}^{-2} \AA^{-1} ;(10,11)$ medium flux in the wavelength range $1420-1520 \AA$ and respective error in units of $10^{-19} \mathrm{erg} \mathrm{s}^{-1} \mathrm{~cm}^{-2} \AA^{-1}$; (12) Ly $\alpha$ equivalent width of the sources that present Ly $\alpha$ in emission. We have indicated the Ly $\alpha$ in absorption with $\geq 0$. We have also marked with * the possible leakers in Sect. 3.2.

We computed the IGM transmissivity following the analytical prescription given by Inoue et al. (2014). They provide a set of analytic functions describing the mean intergalactic attenuation curve for objects at $z>0.5$, simulating, for different lines of sight, a large number of absorbers, and assuming a Poisson probability distribution for a $\mathrm{LyC}$ photon to encounter one of them. The Monte Carlo simulations are based on an empirical distribution function of intergalactic absorbers that they derived from the latest observational statistics of the Ly $\alpha$ forest (LAF), Lyman limit system (LLSs) and damped Ly $\alpha$ systems (DLSs), and is verified to be consistent with the mean Ly $\alpha$ transmission and the mean free path of ionising photons at those redshifts (Worseck et al. 2014). We refer to Inoue et al. (2014) for understanding the limitations and uncertainty related to the application of this prescription. We highlight here that there is a large scatter in the IGM transmission around the mean at each given redshift, as shown, for example, in Fig. 2 of Vanzella et al. (2015).

\subsection{Measuring $f_{\lambda}(895)$ and $f_{\lambda}(1470)$}

For each source in the clean sample, we evaluated from the spectrum the UV flux as the mean value in the wavelength interval $1420-1520 \AA$, and the LyC flux, or a limit, in the range 880-910 $f_{\lambda}(1470)$ and $\left.f_{\lambda}(895)\right)$. We chose the $1420-1520 \AA$ interval for the UV continuum because in this range, no significant spectral features are present. The excellent relative flux calibration adds only a negligible error when computing the $f_{\lambda}(895) / f_{\lambda}(1470)$ ratio, and this is therefore ignored in the following.

We report the individual values in Table 2 with the respective individual statistical errors $\left(\operatorname{err} f_{\lambda}(1470)\right.$ and $\left.\operatorname{err} f_{\lambda}(895)\right)$. As a first approximation, we considered, as non-LyC emitters, all the sources with $f_{\lambda}(895)$ flux consistent with zero within three times their individual statistical error, and consider possible $\mathrm{LyC}$ emitters, as the sources that have values exceeding $3 \times \operatorname{err} f_{\lambda}(895)$ (hereafter referred to as possible leakers). Three of these have a positive $f_{\lambda}(895)$ and one has a negative $f_{\lambda}(895)$. However, the individual errors do not take into account all the possible systematic uncertainties associated, for example, with background subtraction (which change from slit to slit), with the presence of scattered light, or with other quantities that are related to the instrument setup for the masks used. Considering that the objects in our samples were included in many different masks and observed under very different conditions, we decided to assess the 

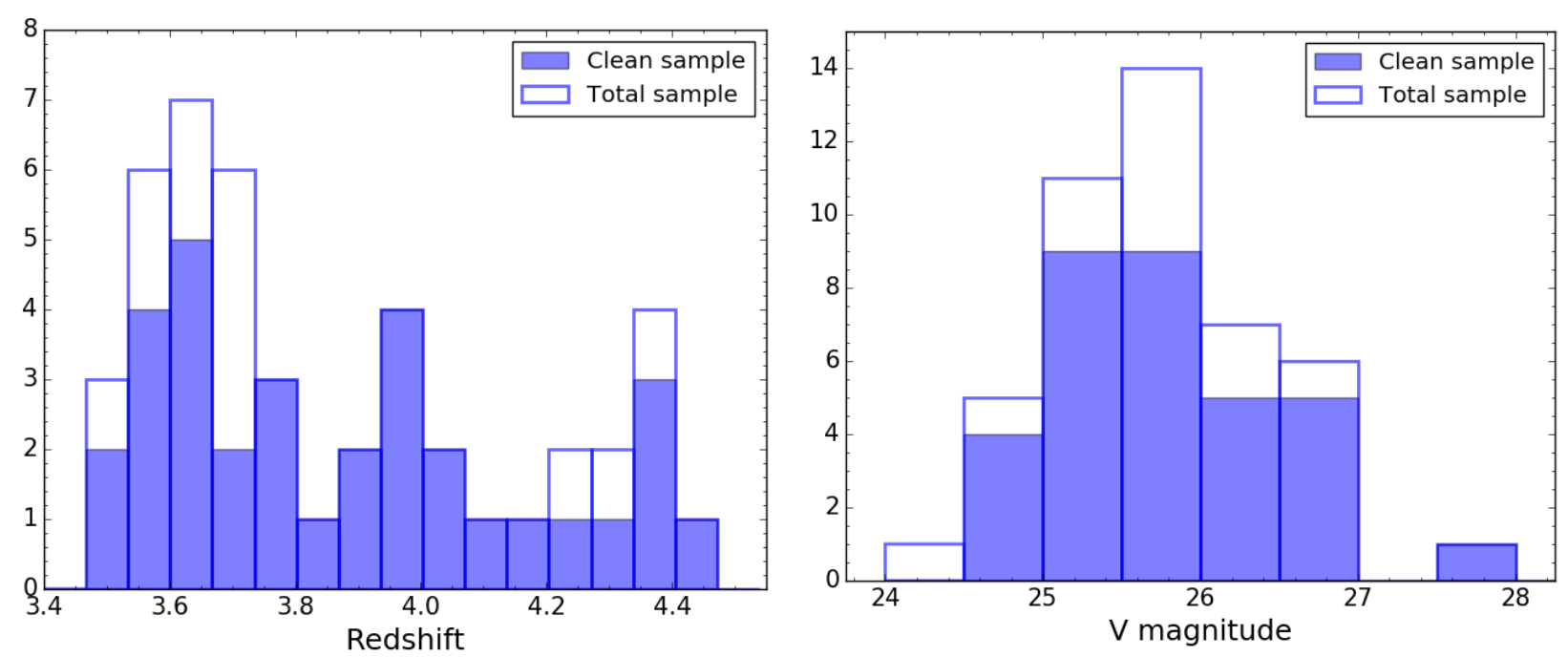

Fig. 3. Left panel: comparison of the redshift distributions of the total sample (empty histogram) and the clean sample (semi-filled histogram). Right panel: comparison of the $V$ magnitude distributions of the total sample (empty histogram) and the clean sample (semi-filled histogram).

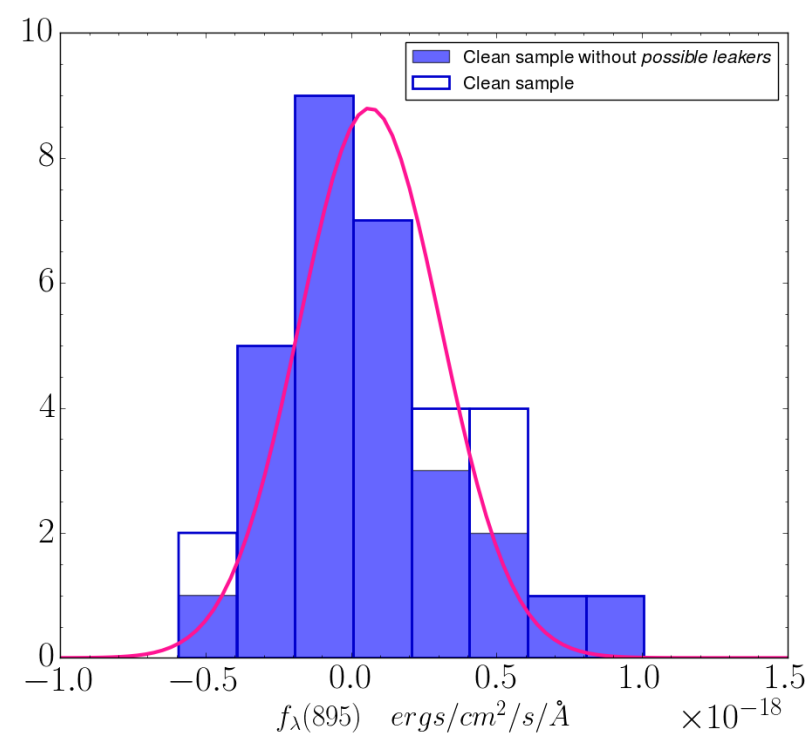

Fig. 4. Distribution of the fluxes measured as the mean fluxes in the $\mathrm{LyC}$ range of each spectrum (880-910 $\AA$ ) for the sources that have LyC flux within three times their standard deviation (filled histogram) and for the clean sample (empty histogram). The Gaussian fit of the distribution without the possible leakers is shown in magenta.

reality of the possible detections by re-evaluating the global error from the distribution of the $f_{\lambda}(895)$ values for the remaining (undetected) objects.

Excluding the possible leakers, we computed the distribution of the LyC fluxes of the non-LyC emitting sources, as shown in Fig. 4; in magenta, we show the Gaussian fit to the distribution that was evaluated after applying a sigma clipping with a limit at $2 \sigma$. This is characterised by a mean value of $f_{\lambda}(\mathrm{LyC})=$ $0.86 \times 10^{-20} \mathrm{erg} \mathrm{s}^{-1} \mathrm{~cm}^{-2} \AA^{-1}$ and a standard deviation of $\sigma_{\mathrm{s}}=2.39 \times 10^{-19} \mathrm{erg} \mathrm{s}^{-1} \mathrm{~cm}^{-2} \AA^{-1}$. The mean value is therefore basically consistent with zero (as expected). On the other hand, the statistical error $\sigma_{\mathrm{s}}=2.39 \times 10^{-19} \mathrm{erg} \mathrm{s}^{-1} \mathrm{~cm}^{-2} \AA^{-1}$ is similar or somewhat larger (by 50\%) than most of the individual errors $f_{\lambda}(895)$ in Table $2\left(\operatorname{err} f_{\lambda}(895)\right.$. We thus assume this value as the mean error in $f_{\lambda}(895)$ for our whole sample; with this assumption, we do not have sources that still show a detection of LyC above $3 \sigma$. All the galaxies in the clean sample therefore have LyC fluxes consistent with zero at $3 \sigma$ level.

\section{Spectral stack}

Since we do not have any solid LyC detection in the individual galaxies, we produced and analysed a stacked spectrum of all sources belonging to the clean sample to increase the sensitivity of our measurement.

To produce the one-dimensional spectral stack, we first shifted each spectrum to its rest-frame using the spectroscopic redshift from VUDS and normalised it using its mean value in the wavelength range 1420-1520 $\AA$ where no particular features are present. To take into account the noise of each spectrum during the stacking procedure, we computed the stack as a weighted average of the spectra in the clean sample using the errors related to the flux density ratio $\frac{f_{\lambda}(895)}{f_{\lambda}(1470)}$ as weights (these had been previously evaluated). We obtained the spectral stack shown in Fig. 5. Note that in this procedure, we do not use precise systemic redshifts, which should be computed only from the interstellar absorption lines or nebular emission lines, and which in many cases cannot be evaluated, but we rely on the VUDS official redshifts (see Le Fèvre et al. 2015, for details on the redshift evaluation). The presence of sharp absorption features in the stack indicates that we can safely use the spectroscopic redshifts in our procedure, since our goal is to analyse the LyC region that covers a broad wavelength range.

We also computed the two-dimensional spectral stack, which is presented in Fig. 5. After shifting each spectrum to the same spatial position (along the slit length) and to its wavelength rest frame, we applied the same weighted average used for the onedimensional stack. The extension of the obtained spectrum is due to the fact that each spectrum has a different spatial width due to different object sizes as projected onto the slit.

\section{Results}

We now derive the average relative escape fraction of the clean sample. The first quantity that is needed is an approximation of the $\frac{f_{v}(895)}{f_{v}(1470)}$ ratio of the clean sample. We evaluate it following two different approaches. The first involves directly measuring 

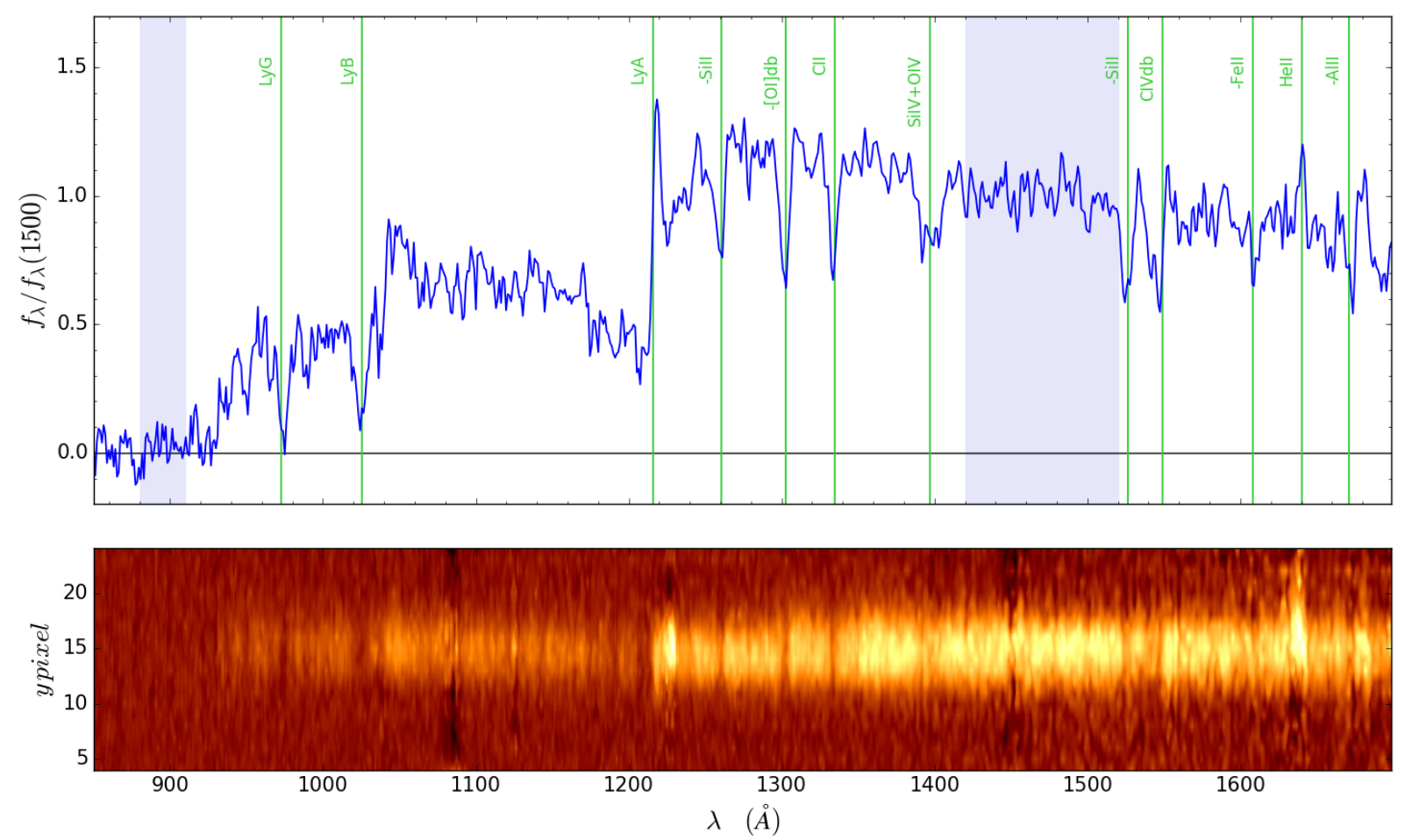

Fig. 5. One-dimensional and two-dimensional spectral stacks of the 33 galaxies in the clean sample. $f_{\lambda} / f_{\lambda}(1470)$ corresponds to the flux normalised at its value at $1470 \AA$. The lavender vertical bands represent the spectral region in which we extracted the LyC flux and the UV flux.

it from the spectral stack (Sect. 4), that is, from the average signal in the wavelength range 880-910 $\AA$. The flux in the spectral stack in Fig. 5 is normalised at the flux value in the range 1420-1520 ̊. We obtain a value of $\frac{f_{v}(895)}{f_{v}(1470)}=0.008 \pm 0.004$. 2) The second method consists of evaluating the flux density ratio for each source in the clean sample and then computing a weighted average using the errors of the ratios that were derived above as weights. With this method, we find $\frac{f_{v}(895)}{f_{v}(1470)}=$ $0.008 \pm 0.005$. Therefore the two methods give almost identical values.

The value of the transmissivity depends on the redshift of the galaxies considered. We proceeded with two different approaches for this quantity as well. The first consists of evaluating the transmissivity for each object in the sample and then averaging them. We call this the mean transmissivity of the sample and we consider this method as the one with a more physical meaning, since it takes into account the uneven redshift distribution of our sample, which is skewed towards the lower redshifts. The second method involves directly evaluating the transmissivity at the median redshift of the clean sample, $z_{\mathrm{MED}}=3.81$. We obtain values of $\mathrm{e}^{-\tau_{\mathrm{IGM}, z}}=0.27$ and $\mathrm{e}^{-\tau_{\mathrm{IGM}, z}}=0.29$, respectively.

Using different combinations of these values, we computed the relative escape fraction of the clean sample. The values that we found are listed in Table 3. The errors are evaluated propagating the error on the flux density ratio to Eq. (1). From the stacked spectrum, we obtain tentative $\geq 2 \sigma$ detections for both the evaluation methods, whereas using the average of individual signals, the overall errors are larger. In all cases, the values are consistent with a null relative escape fraction within $2 \sigma$.

\section{Discussion and future prospects}

Several authors have discussed the possible correlation between the escape of $\mathrm{LyC}$ photons and the escape of $\mathrm{Ly} \alpha$ photons.
Recently, Dijkstra \& Gronke (2016) used a suite of 2500 Ly $\alpha$ Monte-Carlo radiative transfer simulations to show that galaxies with a low $f_{\mathrm{esc}}^{\mathrm{Ly} \alpha}$ consistently have a low $f_{\mathrm{esc}}^{\mathrm{LyC}}$. It is believed that very compact galaxies with a strong Ly $\alpha$ in emission are the most plausible candidates to show LyC leakage. Bogosavljevìc (2010) found that their LyC-detected sample showed a significantly stronger emission of Ly $\alpha$ photons on average, with respect to the non-detected sample, although their sample was prone to low redshift contamination since it lacked high-resolution HST imaging. Finally, an empirical correlation between EW(Ly $\alpha)$ and $f_{\text {esc }}($ LyC) has also been shown by Verhamme et al. (2017) for the small number of LyC confirmed sources, including both local galaxies and Ion2 (Vanzella et al. 2015).

We therefore investigated whether or not the limits on the escape fraction of the individual sources in our sample have any correlation to the presence of $\operatorname{Ly} \alpha$. For each spectrum that shows Ly $\alpha$ in emission, we measured the equivalent width (EW) of the line using IRAF. We report the values of the EW of the sources in our sample in Table 2 where we have only indicated $E W(\operatorname{Ly} \alpha) \geq 0$ for the spectra that show the line in absorption. We compared the EW derived with this method to those derived by Cassata et al. (2015) and found, in general, a very good agreement.

In Fig. 6, we show the flux density ratio as a function of the absolute value of the rest frame equivalent width of the Ly $\alpha$, for the sources that have the line in emission. The errors on the flux density ratios have been evaluated using the individual errors of each object. Despite the fact that we have few Ly $\alpha$ emitters in our sample, from the figure, we see indications of a possible trend of the flux density ratio as a function of $\operatorname{Ly} \alpha \mathrm{EW}$, in the sense that, if Ly $\alpha$ is in emission, the ratio increases with increasing EW. This trend could therefore indicate that the mechanisms that drive the escape of Ly $\alpha$ photons also facilitate the escape of LyC radiation. The same tentative trend is found by Micheva et al. (2015) with a sample of 18 LAEs at $z \geq 3$.06. In particular, we point out that 
Table 3. Relative escape fraction of the clean sample for the different combinations of transmissivities and $\frac{f_{v}(895)}{f_{v}(1470)}$ ratios that we found with the different methods explained in Sect. 5.

\begin{tabular}{lcc}
\hline \hline & Mean transmissivity & Transmissivity from the median redshift \\
\hline Flux ratio from the stack & $f_{\mathrm{esc}}^{\mathrm{rel}}=0.09 \pm 0.04$ & $f_{\mathrm{esc}}^{\mathrm{rel}}=0.08 \pm 0.04$ \\
Flux ratio from the average & $f_{\mathrm{esc}}^{\mathrm{rel}}=0.09 \pm 0.06$ & $f_{\mathrm{esc}}^{\mathrm{rel}}=0.08 \pm 0.05$ \\
\hline
\end{tabular}

the only two sources in the clean sample that show a very strong Ly $\alpha$ emission, have a high flux density ratio with respect to the average of the other sources (they are the two upper right points in Fig. 6). In addition, both have a very compact morphology, as shown in Fig. 7, so they would be good LyC emitter candidates according to Izotov et al. (2016), who show that selecting compact star-forming galaxies with high [OIII] $] 25007 /[\mathrm{OII}] \lambda 3727 \mathrm{ra}-$ tios appears to very efficiently pick up sources with escaping Lyman continuum radiation. In particular, one of the our two sources, namely VUDS ID 530075924, was identified as a possible emitter in the initial screening of the sample; when considering its individual statistical errors, the LyC flux was detected with a $>4 \sigma$ significance, and it was therefore included in the possible leakers sample (see Sect. 3.2). However, after the re-evaluation of the error from the sample distribution, the significance of the detection dropped to $2.5 \sigma$ and it was therefore considered consistent with a non-emitter. Given the high flux density ratio, the large Ly $\alpha$ EW and the compact morphology, this galaxy could, in fact, be considered our best candidate LyC leaker. In addition, this object also has the same properties of the galaxies described in Amorin et al. (in prep.), which are selected to be extremely young (metal-poor) dwarf galaxies, typical features of LyC leakers at low redshift. Taking the LyC flux at face value and the mean transmissivity at that redshift, this source would have a relative escape fraction $>100 \%$ with a very large uncertainty. This extremely high value could only be considered physical in the specific cases in which the galaxy resides in a region with a clearer line-of sight (thus with higher transmissivity) compared to the average, or if the galaxy had a smaller intrinsic $L_{v}(1470) / L_{v}(895)$ than the standard value assumed. Alternatively, it could be characterised by a different dust geometry, as in the case of runaway massive stars that emit LyC radiation near the border of the galaxy where the coverage of the dust is less effective (Conroy \& Kratter 2012). We plan to further investigate the nature of this source, however, we note that to eventually confirm its nature as a LyC leaker would require a significant amount of integration time with currently existing instrumentation, given that the VUDS spectrum is already the result of $14 \mathrm{~h}$ integration on the VLT.

We finally checked if the limits on the escape fraction of individual sources have any correlation to the UV magnitude but no trend is observed in agreement with what has previously been found; by Guaita et al. (2016), for example.

In conclusion, in this study, we do not find any solid individual detection of LyC emission, although we have a possible interesting candidate, discussed above. We obtain an average relative escape fraction of the clean sample of $f_{\mathrm{esc}}^{\mathrm{rel}}=$ $0.09 \pm 0.04$ by stacking all the individual spectra. This tentative detection is consistent with what we found in our previous study (Guaita et al. 2016) based on a similar clean sample, that is, not effected by low-redshift contamination, and studied through narrow band photometry, for which we reported a $f_{\text {esc }}^{\text {rel }}<12 \%$. This value is also consistent with what has found previously by Grazian et al. (2016) and Boutsia et al. (2011) at a similar redshift. This is also in agreement with Vanzella et al. (2010).

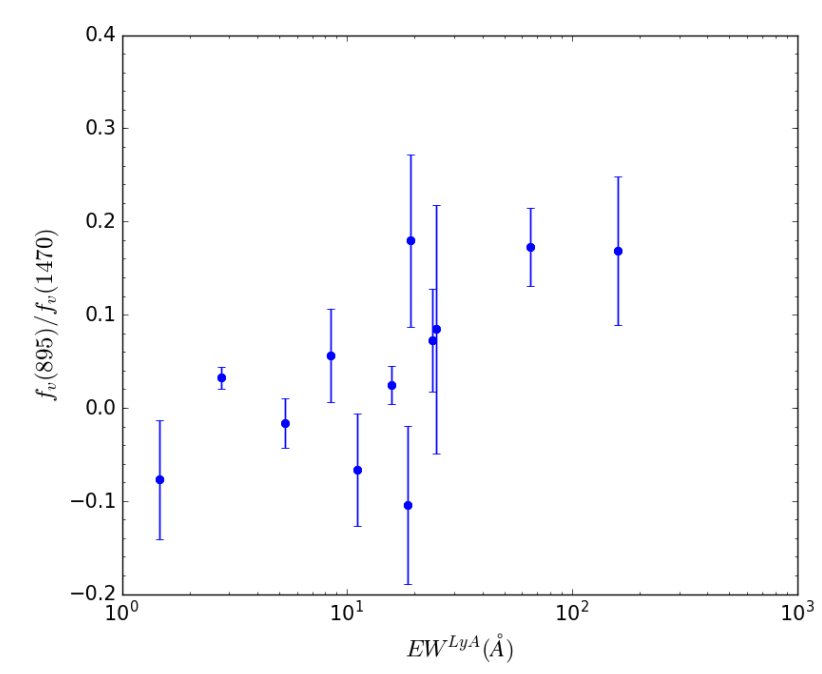

Fig. 6. Ratio between the mean fluxes at $895 \AA$ and $1470 \AA$ in units of ergs s $\mathrm{cm}^{-1} \mathrm{~Hz}^{-1}$ as a function of the absolute value of the rest frame equivalent width of the Ly $\alpha$ for the 12 galaxies that have Ly $\alpha$ in emission.
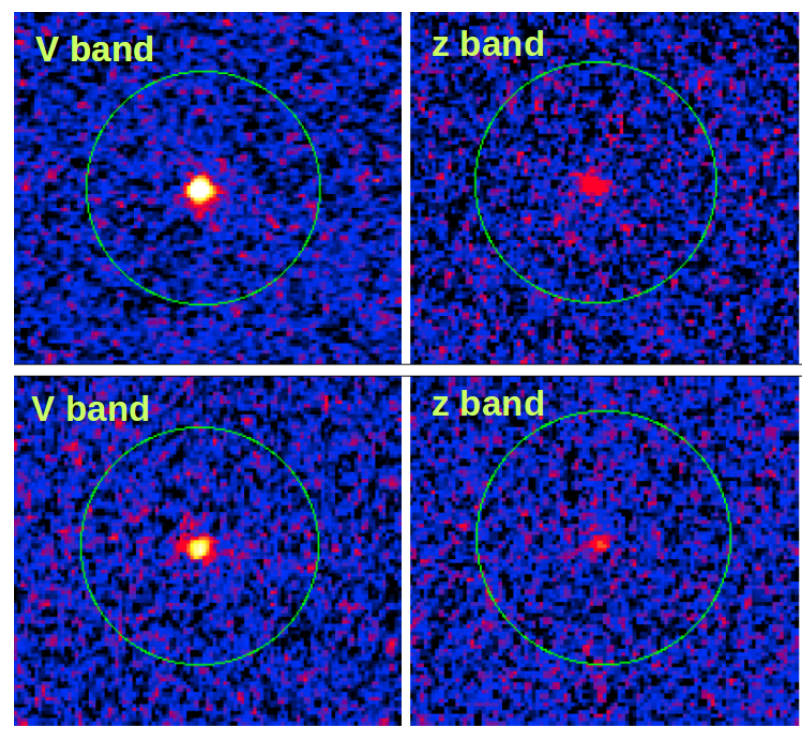

Fig. 7. GEMS images in the $V$ and $z$ band of the two sources: 530075924 (upper panel) and 535003653 (bottom panel). The circular regions have 1 arcsec radius.

They used ultra-deep ultraviolet VLT/VIMOS intermediate-band and VLT/FORS1 narrow-band imaging in the GOODS-S field to derive limits on the distribution of the absolute escape fraction for LBGs in the redshift interval 3.4-4.5. They found a median $f_{\text {esc }}$ lower than $\sim 6 \%$ with an $84 \%$ percentile limit not larger than

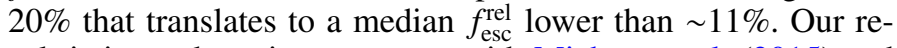
sult is instead not in agreement with Micheva et al. (2015) and Smith et al. (2016). Micheva et al. (2015) analysed samples of 18 LAEs and 7 LBGs at $z \geq 3.06$ obtained from the SSA22 field with Subaru/Suprime-cam. They found values for the relative 
escape fraction of $\sim 30 \%$ for the LAEs sample and $\sim 20 \%$ for the LBGs sample. These high values could however be due, in part, to foreground contamination, since many LyC emitter candidates in their samples show a spatial offset between the rest-frame UV and LyC emissions. Contamination from lower redshift interlopers could also be present in Smith et al. (2016) who presented observations of escaping LyC radiation from 50 galaxies in different redshift bins from $z \sim 2.3$ to $z \sim 5.8$ in the Early Release Science (ERS) field. They found $f_{\mathrm{esc}}^{\mathrm{rel}}=19.8_{-10.6}^{+39.2}$ for galaxies at $z \sim 3.5$; at $z \sim 5$ however the relative escape fraction exceeds $100 \%$, indicating a possible contamination effect.

We plan to apply the same method to another upcoming spectroscopic survey, VANDELS, ${ }^{2}$ that will give us, in one year, spectra of high-redshift galaxies with unprecedented observation time of up to $80 \mathrm{~h}$, also with VIMOS. With these spectra, we might be able to detect LyC emitters or set much stronger constraints on the average escape fraction of high-redshift galaxies. In particular, by using the target catalogues in the CDFS and UDS fields and the relative photometric redshifts, we have estimated that we will be able to study between 100-150 LBGs and star-forming galaxies at $4.4 \leqslant z_{\text {phot }} \leqslant 5.0$ where the LyC range is still included in the VANDELS spectra. Comparing our predictions with the sensitivity of the instrumentation, we have estimated that we will be able to detect the LyC in individual LBGs if $f_{\mathrm{esc}}^{\mathrm{rel}}>0.05-0.2$ and average signals of $f_{\mathrm{esc}}^{\mathrm{rel}} \sim 0.02-0.05$ from the stacks of 30-50 objects samples, thus setting much tighter constraints on the properties of high-redshift galaxies.

Acknowledgements. We thank the ESO staff for their continuous support for the VUDS survey, particularly the Paranal staff conducting the observations and Marina Rejkuba and the ESO user support group in Garching. This work is supported by funding from the European Research Council Advanced Grant ERC2010-AdG-268107-EARLY and by INAF Grants PRIN 2010, PRIN 2012 and PICS 2013. A.C., O.C., M.T. and V.S. acknowledge the grant MIUR PRIN 20102011. This work is based on data products made available at the CESAM data center, Laboratoire d'Astrophysique de Marseille. R.A. acknowledges support from the ERC Advanced Grant 695671 "QUENCH".

\section{References}

Bertin, E., \& Arnouts, S. 2010, Astrophysics Source Code Library [record ascl: 1010.064]
Bogosavljevìc, M. 2010, Ph.D. Thesis, California Institute of Technology, USA Boutsia, K., Grazian, A., Giallongo, E., et al. 2011, ApJ, 736, 41 Bruzual, G., \& Charlot, S. 2003, MNRAS, 344, 1000

Cardamone, C. N., van Dokkum, P. G., Urry, C. M., et al. 2010, ApJS, 189, 270 Cassata, P., Tasca, L. A. M., Le Fèvre, O., et al. 2015, A\&A, 573, A24 Chabrier, G. 2003, PASP, 115, 763

Conroy, C., \& Kratter, K. M. 2012, ApJ, 755, 123

Dahlen, T., Mobasher, B., Faber, S. M., et al. 2013, ApJ, 775, 93

de Barros, S., Vanzella, E., Amorín, R., et al. 2016, A\&A, 585, A51

Dijkstra, M., \& Gronke, M. 2016, ApJ, 828, 71

Giallongo, E., Cristiani, S., D’Odorico, S., \& Fontana, A. 2002, ApJ, 568, L9

Giallongo, E., Grazian, A., Fiore, F., et al. 2015, A\&A, 578, A83

Grazian, A., Fontana, A., Santini, P., et al. 2015, A\&A, 575, A96

Grazian, A., Giallongo, E., Gerbasi, R., et al. 2016, A\&A, 585, A48

Grogin, N. A., Kocevski, D. D., Faber, S. M., et al. 2011, ApJS, 197, 35

Guaita, L., Pentericci, L., Grazian, A., et al. 2016, A\&A, 587, A133

Inoue, A. K., Shimizu, I., Iwata, I., \& Tanaka, M. 2014, MNRAS, 442, 1805

Iwata, I., Inoue, A. K., Matsuda, Y., et al. 2009, ApJ, 692, 1287

Izotov, Y. I., Schaerer, D., Thuan, T. X., et al. 2016, MNRAS, 461, 3683

Koekemoer, A. M., Faber, S. M., Ferguson, H. C., et al. 2011, ApJS, 197, 36

Le Fèvre, O., Tasca, L. A. M., Cassata, P., et al. 2015, A\&A, 576, A79

Madau, P. 1995, ApJ, 441, 18

Micheva, G., Iwata, I., Inoue, A. K., et al. 2015, ArXiv e-prints [arXiv: 1509.03996]

Mostardi, R. E., Shapley, A. E., Nestor, D. B., et al. 2013, ApJ, 779, 65 Mostardi, R. E., Shapley, A. E., Steidel, C. C., et al. 2015, ApJ, 810, 107 Nestor, D. B., Shapley, A. E., Steidel, C. C., \& Siana, B. 2011, ApJ, 736, 18 Rix, H.-W., Barden, M., Beckwith, S. V. W., et al. 2004, ApJS, 152, 163 Robertson, B. E., Ellis, R. S., Furlanetto, S. R., \& Dunlop, J. S. 2015, ApJ, 802, L19

Santini, P., Ferguson, H. C., Fontana, A., et al. 2015, ApJ, 801, 97

Shapley, A. E., Steidel, C. C., Pettini, M., Adelberger, K. L., \& Erb, D. K. 2006, ApJ, 651, 688

Shapley, A. E., Steidel, C. C., Strom, A. L., et al. 2016, ApJ, 826, L24

Siana, B., Teplitz, H. I., Colbert, J., et al. 2007, ApJ, 668, 62

Siana, B., Shapley, A. E., Kulas, K. R., et al. 2015, ApJ, 804, 17

Smith, B. M., Windhorst, R. A., Jansen, R. A., et al. 2016, ApJ, submitted, [arXiv: 1602.01555]

Steidel, C. C., Pettini, M., \& Adelberger, K. L. 2001, ApJ, 546, 665

Thomas, R., Le Fèvre, O., Le Brun, V., et al., 2017, A\&A, 597, A88

Vanzella, E., Giavalisco, M., Inoue, A. K., et al. 2010, ApJ, 725, 1011

Vanzella, E., Guo, Y., Giavalisco, M., et al. 2012, ApJ, 751, 70

Vanzella, E., de Barros, S., Castellano, M., et al. 2015, A\&A, 576, A116

Vanzella, E., de Barros, S., Vasei, K., et al. 2016, ApJ, 825, 41

Verhamme, A., Orlitova, I., Schaerer, D., et al. 2017, A\&A, 597, A13

Wise, J. H., \& Cen, R. 2009, ApJ, 693, 984

Worseck, G., Prochaska, J. X., O'Meara, J. M., et al. 2014, MNRAS, 445, 1745

Wyithe, J. S. B., \& Cen, R. 2007, ApJ, 659, 890

2 http://vandels.inaf.it/ 


\section{Appendix A: The importance of the cleaning procedure}

We show in Fig. A.1 the comparison between the stack of the clean sample (blue spectrum) and the stack of the total sample (magenta spectrum) in order to point out the importance of the cleaning procedure in these kind of studies. The flux of the total sample is indeed a factor of 5 greater than the flux of the clean sample in the LyC region (turquoise vertical band in Fig. A.1) whereas they have the same values in the UV part of the spectrum. This exceeding flux is evidence of the contamination from lower redshift interlopers that we avoided thanks to the cleaning procedure.

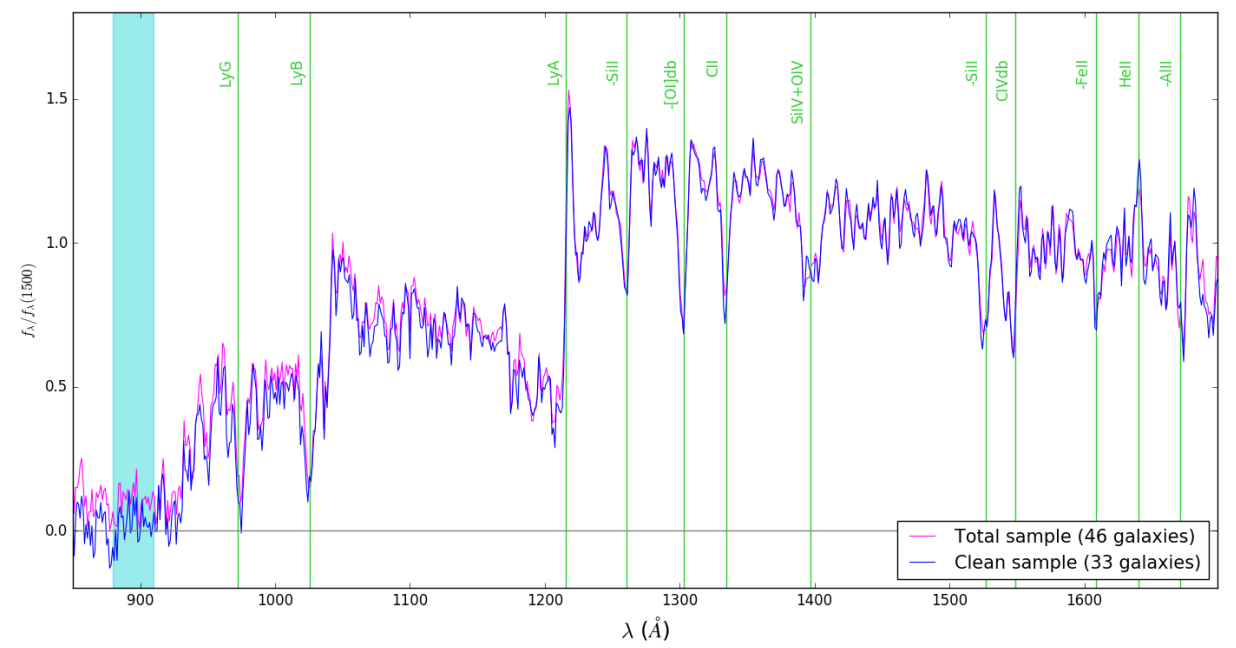

Fig. A.1. One-dimensional stack of the clean sample (blue line) compared with the stack of the total sample before the application of the cleaning procedure (magenta line). 